\title{
A directed search algorithm for setting the spectral-spatial quality trade-off of fused images by the wavelet à trous method
}

\author{
Consuelo Gonzalo and Mario Lillo-Saavedra
}

\begin{abstract}
This paper proposes a method to determine, in an objective and accurate way, the weighting factor $(\alpha)$ to be applied to the detailed panchromatic image information that will be integrated with the background multispectral image information to obtain the "best" fused image with the same spatial and spectral quality. The fusion method is a weighting variant of the fusion algorithm based on the wavelet transform, calculated using the à trous (WAT) algorithm. The $\alpha$ factor is determined, for each band of the multispectral source images using the simulated annealing (SA) search algorithm, which optimizes an objective function (OF) associated with both spatial and spectral quality measures for the fused images. The results obtained have demonstrated that for each one of the spectral bands there is an $\alpha$ value that provides fused images with the optimal trade-off between the two qualities for any decomposition level value $(n)$ of the wavelet transform.
\end{abstract}

Résumé. Dans cet article, on propose une méthode pour déterminer, de façon objective et précise, le facteur de pondération $(\alpha)$ à appliquer à l'information détaillée d'une image panchromatique qui sera intégrée à l'information de l'image multispectrale de base afin d'obtenir la «meilleure » image fusionnée avec la même qualité spatiale et spectrale. La méthode de fusion est une variante de pondération de l'algorithme de fusion basé sur la transformée en ondelettes, calculée à l'aide de l'algorithme à trous. Le facteur $\alpha$ est déterminé, pour chaque bande des images multispectrales sources, en utilisant l'algorithme de recherche du recuit simulé (SA), qui optimise la fonction objectif (FO) associée avec les mesures de la qualité spatiale et spectrale des images fusionnées. Les résultats obtenus ont démontré que, pour chacune des bandes spectrales, il y a une valeur de $\alpha$ qui fournit aux images fusionnées un compromis optimal entre les deux qualités pour toute valeur de niveau de décomposition $(n)$ de la transformée en ondelettes.

[Traduit par la Rédaction]

\section{Introduction}

Among multiresolution image fusion strategies, the wavelet transform calculated using the Mallat pyramidal algorithm (wavelet Mallat transform, WMT) (Mallat, 1999) is one of the most frequently used because of the high spectral quality characteristic of the resulting images. Still, its low anisotropy provokes problems when fusing images with a large number of borders in directions that are not horizontal, vertical, or diagonal (Núñez et al., 1999; Candès and Donoho, 2000; González-Audicana et al., 2005).

In 1987, Dutilleux proposed the wavelet transform calculated using the à trous ("with holes") algorithm (wavelet à trous transform, WAT). This algorithm presents two fundamental differences compared to pyramidal-type algorithms: WAT has an isotropic nature and it is redundant in the sense that, between two consecutive degradation levels, there is no dyadic spatial compression of the original image, but rather it maintains the original size of the image.
A comparative study presented in the literature (Chibani and Houacine, 2003; González-Audicana et al., 2005) has shown that spatial quality of the images fused using the WAT method is superior to that provided by the WMT method. For both methods, there is a wide variety of strategies that can be used to integrate the spatial information contained in the panchromatic image (PAN) into each one of the bands of the multispectral image (MULTI) (Núñez et al., 1999).

The need to establish a trade-off between the spectral and spatial quality of the fused images has been noted, and different approaches have been proposed for controlling the quality. Some approaches have proposed an external mechanism to the fusion method (Lillo-Saavedra et al., 2005; Yunhao et al., 2006), and others have introduced modifications to known methods (LilloSaavedra and Gonzalo, 2006a; Choi, 2006; Tu et al., 2007). New strategies that intrinsically provide this control have been proposed (Choi et al., 2005; Lillo-Saavedra and Gonzalo, 2007). The main problem with all of these strategies is the determination of parameters involved in the control process.

C. Gonzalo. ${ }^{1}$ Departamento de Aquitectura y Tecnología de Sistemas Informáticos, Facultad de Informática, Universidad Politécnica de Madrid, Campus de Montegancedo, Boadilla del Monte, 28660 Madrid, Spain.

M. Lillo-Saavedra. Faculty of Agricultural Engineering, University of Concepción, Av. Vicente Méndez 595 Casilla, 537 Chillán, Chile.

${ }^{1}$ Corresponding author (e-mail: consuelo.gonzalo@upm.es). 
This work has been motivated by the weighting WAT fusion method proposed in Lillo-Saavedra and Gonzalo (2006a). The goal of this method is to establish the degree of trade-off between spectral and spatial quality of the fused image through the characteristic curves. These curves represent spatial and spectral quality indices in the same domain, meaning that fused images with different characteristics can be obtained when the weighting factor $(\alpha)$ varies (see the next section).

The present paper proposes a method to objectively determine the weighting factor $(\alpha)$ of the fusion algorithm proposed in Lillo-Saavedra and Gonzalo (2006a) using the simulated annealing (SA) search algorithm. This optimizes an objective function (OF) associated with both spatial and spectral quality measures of the fused image, which allows a directed search of the optimal solution. The proposed fusion method has been called WATSA.

\section{Fusion methodology based on the wavelet à trous transform (WAT)}

From a practical point of view, WAT consists basically of successive convolutions between the image to be analyzed and a low-pass filter called the "scaling function." One of the most used scaling functions is the $b_{3}$-spline, which can be represented by the following equation:

$h_{j}=\frac{1}{256}\left(\begin{array}{ccccc}1 & 4 & 6 & 4 & 1 \\ 4 & 16 & 24 & 16 & 4 \\ 6 & 24 & 36 & 24 & 6 \\ 4 & 16 & 24 & 16 & 4 \\ 1 & 4 & 6 & 4 & 1\end{array}\right)$

The filter to be applied in the next decomposition level is obtained from the filter applied in the previous level, intercalating it with zeros in the rows and columns.

The wavelet coefficients $\left(C_{j}\right)$ are obtained from the difference between two consecutive decomposition levels as shown in the following equation:

$C_{j+1}(x, y)=I_{j}(x, y)-I_{j+1}(x, y)$

To synthesize the image from a decomposition level $j+n$, an additive criterion that adds all the coefficients obtained to the last decomposition level can be applied, as shown in the following equation:

$I_{j}(x, y)=I_{j+n}(x, y)+\sum_{k=1}^{n} C_{j+k}(x, y)$

where $I_{j+n}(x, y)$ represents a background image that contains low-frequency information of the original image; and $C_{j+n}(x, y)$ represents their respective wavelet coefficients, which contain high-frequency information. From Equations (2) and (3), an image fusion strategy can be proposed, in which the low- frequency information contained in a MULTI image can be integrated with the high-frequency information contained in the wavelet coefficients of a high-resolution spatial image (PAN), resulting in a multispectral image with high spatial resolution. This fusion strategy does not provide control on the spatial and spectral quality of the fused images. In this sense, a weighting WAT version of a fusion strategy, based on the a trous algorithm that introduces an objective trade-off criterion between spectral and spatial quality of the fused images, has been proposed in Lillo-Saavedra and Gonzalo (2006a) as follows:

$I_{\mathrm{FUS}^{i}}^{i}(x, y)=I_{\mathrm{MULTI}_{j+n}}^{i}(x, y)+\alpha^{i} \sum_{k=1}^{n} C_{\mathrm{PAN}_{j+k}}^{i}(x, y)$

where the superscript $i$ represents the $i$ th spectral bands of the MULTI image; $n$ is the number of decompositions levels from the $j$ level; $\sum_{k=1}^{n} C_{\text {PAN }_{k}}^{i}$ represents the sum of all PAN image wavelet coefficients; and $\alpha^{i}$ represents the weighting factors of these coefficients for each band. Figure 1 presents a scheme of the fusion procedure.

It has been proven that there is a direct relation between the quality of the fused images and the number of decomposition levels of the source images (Lillo-Saavedra and Gonzalo, 2006b). This implies the determination of the number of wavelet PAN coefficients that should be integrated in the $n$th decomposition level of the MULTI to obtain the best spatialspectral quality for each set of images to be fused.

\section{Determination of the weighting factor}

Lillo-Saavedra and Gonzalo (2006a) proposed determining the weighting factor of the PAN image wavelet coefficients by searching for the best trade-off between the spectral and spatial quality of the fused image. The spectral and spatial differences between the source images and the fused image were measured by means of two indices, namely the original "erreur relative globale adimensionalle de synthèse" (ERGAS; Wald, 2002), which is referred to in this paper as spectral ERGAS $\left(\right.$ ERGAS $\left._{\text {spectral }}\right)$, and the spatial ERGAS (ERGAS spatial $)$ (LilloSaavedra and Gonzalo, 2005).

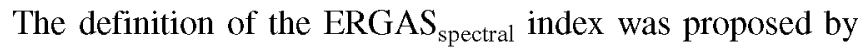
Wald (2002) through the following equation:

ERGAS $_{\text {spectral }}=100 \frac{h}{l} \sqrt{\frac{1}{N_{\text {Bands }}} \sum_{i=1}^{N_{\text {Bands }}}\left[\frac{\operatorname{RMSE}_{\text {spectral }}^{2}\left(\text { Band }_{i}\right)}{\left(M_{\text {MULTI }}^{i}\right)^{2}}\right]}$

where $h$ and $l$ represent the spatial resolution of the PAN and MULTI images, respectively; $N_{\text {Bands }}$ is the number of bands of the fused image; $M_{\text {MULTI }}^{i}$ is the mean radiance value of the $i$ th band of the MULTI image; and, from original the definition

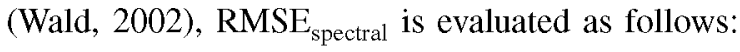




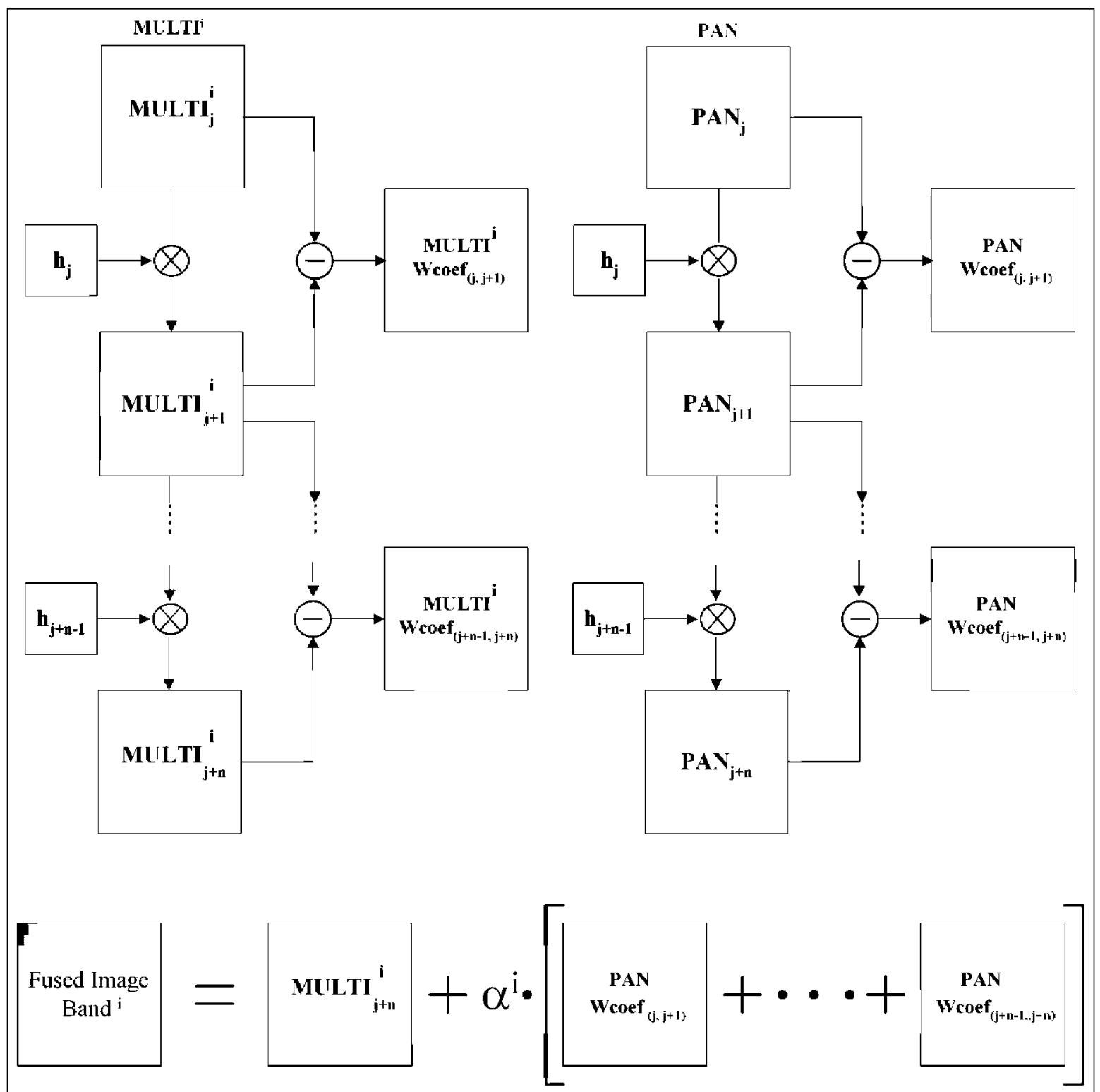

Figure 1. Diagram of the weighted WAT fusion method.

$\operatorname{RMSE}_{\text {spectral }}\left(\operatorname{Band}_{i}\right)=\frac{1}{\mathrm{NP}} \sqrt{\sum_{k=1}^{\mathrm{NP}}\left[I_{\mathrm{REF}}^{i}(k)-I_{\mathrm{FUS}}^{i}(k)\right]^{2}}$

where NP is the number of pixels of the fused image; $I_{\mathrm{REF}}^{i}$ represents the reference multispectral image; and $I_{\mathrm{FUS}}^{i}$ represents the fused image. It is clear from its definition that low ERGAS index values represent the high quality of the fused images.

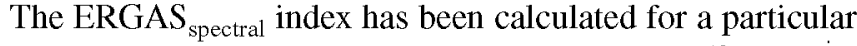
image and different values of the weighting factor $\left(0 \% \leq \alpha^{i} \leq\right.$ $200 \%$ ), and the resulting curve is shown in Figure 2. This curve is at zero when $\alpha^{i}=0 \%$ (no PAN information is injected into the fused image), and the index values rise as $\alpha^{i}$ increases, which means that the injection of spatial information from the PAN image increases the spectral difference between the original

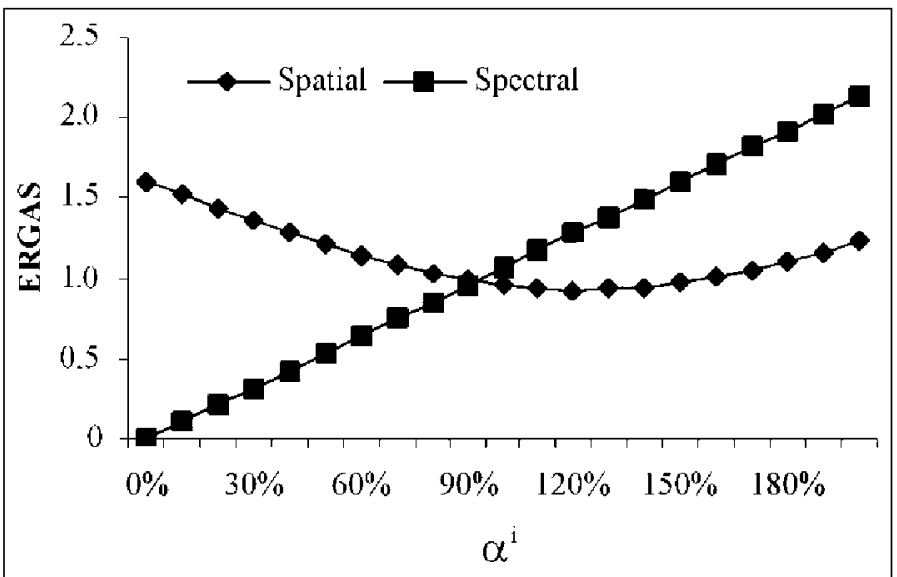

Figure 2. Graphical determination of the weighting factor $\alpha^{i}$. 
MULTI image and the fused image (FUS); however, spatial differences between the PAN image and the FUS image should decrease. In this sense, Lillo-Saavedra et al. (2005) proposed a new index with the objective of evaluating the distance between the PAN image and the FUS image (spatial quality). This index has been called spatial ERGAS (ERGAS spatial $_{\text {) }}$ ) because it is based on the same concept as that of the original ERGAS $_{\text {spectral }}$ (Wald, 2002). In its definition, a spatial RMSE has been included, which is defined as in the following equation:

$\operatorname{RMSE}_{\text {spatial }}\left(\operatorname{Band}_{i}\right)=\frac{1}{\mathrm{NP}} \sqrt{\sum_{k=1}^{\mathrm{NP}}\left[I_{\mathrm{PAN}}^{i}(k)-I_{\mathrm{FUS}}^{i}(k)\right]^{2}}$

where $I_{\text {PAN }}^{i}$ is the image obtained by adjusting the histogram of the original PAN image to the histogram of the $i$ th band of the FUS image. In this way the spectral differences between the PAN and fused images are minimized. Therefore, the following expression is obtained by replacing $M_{\text {MULTI }}^{i}$ with $M_{\mathrm{PAN}}^{i}$ in Equation (5):

ERGAS $_{\text {spatial }}=100 \frac{h}{l} \sqrt{\frac{1}{N_{\text {Bands }}} \sum_{i=1}^{N_{\text {Bads }}}\left[\frac{\operatorname{RMSE}_{\text {spatial }}^{2}\left(\mathrm{Band}_{i}\right)}{\left(M_{\mathrm{PAN}}^{i}\right)^{2}}\right]}$

Figure 2 illustrates the behavior of the spatial ERGAS. As $\alpha^{i}$ increases, or in other words the spatial content increases, the corresponding curve tends to zero.

The availability of these two indices, namely spatial and spectral ERGAS, that describe the spatial and spectral quality behavior of the fused images independently, but within the same domain and without saturation effects (see Figure 2), permitted the definition of a trade-off between both qualities in a natural way in Lillo-Saavedra and Gonzalo (2006a). Figure 2 illustrates the graphical process for obtaining the weighting factor of the PAN wavelet coefficients. This process implies the fusion of an elevated number of images, and therefore it is a costly and imprecise fusion strategy from an operational point of view.

\section{Search algorithm}

The potentiality of the weighted WAT fusion methodology is be strengthened with a procedure to quickly and accurately determine the $\alpha^{i}$ values that optimize the trade-off between spatial and spectral quality of fused images.

The stochastic search method called simulated annealing (SA) is proposed in this paper to determine these parameters. To establish the aforementioned trade-off, a fitness function has been defined as the difference between the spatial and spectral ERGAS indices $(\Delta E)$ as formalized in the following equation:

$\Delta E=\mid$ ERGAS $_{\text {spatial }}-$ ERGAS $_{\text {spectral }} \mid$
The principles of the SA optimization method developed by Kirkpatrick et al. (1983) are based on the physical analogy with the behavior of a set of atom nuclei, approximating to the thermodynamic equilibrium at a determined temperature, understanding a thermodynamic equilibrium as that state in which there is no energetic exchange between system components.

The SA searches a new solution that lies in the vicinity of the actual solution every time the process iterates. The difference between the objective functions (OF) associated with both solutions is then calculated. If the difference is less than a certain threshold, then the new solution becomes the actual solution and the process is repeated.

In the SA algorithm is necessary a random variable that follows a certain probability function with values between zero and infinity. The acceptance of worse solutions is governed by the following criterion:

$\operatorname{rand}(0,1)<\exp \left\{\left[\mathrm{OF}\left(x_{i+1}\right)-\mathrm{OF}\left(x_{i}\right)\right] / T\right\}$

where $T$ represents a parameter called "temperature," and $\operatorname{rand}(0,1)$ is a random number between 0 and 1 with an uniform probability distribution. Figure 3 presents graphically the methodology used to search the value of $\alpha^{i}$ for each spectral band. As can be seen, once the preprocessing of the source images is complete, an initial value is established as $\alpha_{\text {ini }}^{i}=100 \%$. In other words, the process starts from a first fused image obtained by the standard WAT fusion methodology. The spectral and spatial ERGAS quality indices of this initial image will be determined to calculate the initial value of the objective function (Equation (9)).

The study of the behavior of the ERGAS indices with respect to the variation of the parameter $\alpha^{i}$ showed that an increase in the parameter diminishes the fused image's spectral quality while increasing its spatial quality, and vice versa (Figure 2). The oriented search criterion was established based on this behavior: if the value of $\Delta E_{\mathrm{ini}}$ is less than zero $\left(\mathrm{ERGAS}_{\mathrm{spatial}}<\right.$ ERGAS $_{\text {spectral }}$ ), then the spectral quality should improve as the spatial quality of the fused image decreases. Consequently, the value of the parameter $\alpha_{\mathrm{ini}}^{i}$ should decrease in a value $\mathrm{d} \alpha$. In the opposite case, for $\Delta E_{\text {ini }}$ greater than zero, the spatial quality of the fused image should increase, implying an increase of $\mathrm{d} \alpha$ in the value of the parameter $\alpha_{\text {ini }}^{i}$. The value of $\mathrm{d} \alpha$ has been calculated as shown in the following equation:

$\mathrm{d} \alpha=\left|\Delta E_{\text {ini }}\right| \operatorname{rand}(0,1)$

As can be observed in Equation (11), d $\alpha$ takes random values scaled in the $\Delta E_{\text {ini }}$ range, which decreases with the algorithm convergence, being the changes of $\mathrm{d} \alpha$ finer. Once the new solution $\Delta E_{\text {end }}$ is obtained from the new parameter $\alpha_{\text {end }}^{i}=\alpha_{\text {ini }}^{i} \pm \mathrm{d} \alpha$, this will be accepted and discarded according to the SA algorithm acceptance criterion described at the beginning of this section. Thus, in each iteration a new fused image is obtained by the methodology formalized in Equation (4), with its ERGAS index values calculated an a new 


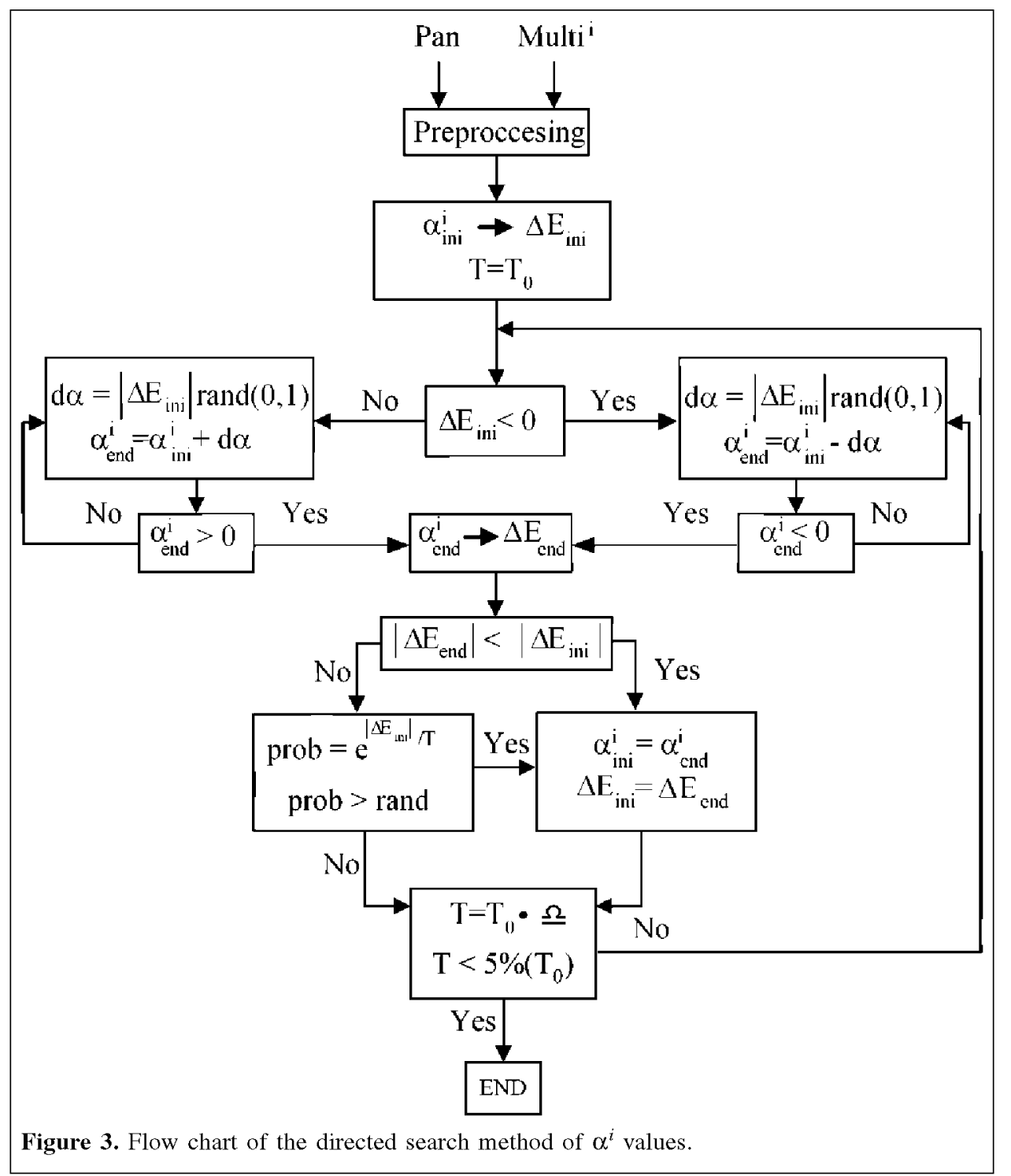

value of the OF ( $\left.\Delta E_{\text {end }}\right)$ determined to go to the next SA iteration.

The SA strategy is to begin with a "high" initial temperature, which provides a high probability of accepting worse solutions. For each iteration, the temperature is reduced, consequently diminishing the probability of accepting the solutions. This temperature-reduction process is known as the cooling schedule and is controlled by a temperature-decreasing index ( $\delta$ ). A very small value of $\delta$ will imply a rapid convergence of the algorithm. However, this would result in a barely exhaustive search, which would increase the probability of being trapped at local minima. In contrast, at a high value of $\delta$, the search algorithm will slowly converge because it is more exploratory, increasing the probability of obtaining solutions closer to the global minimum.

Joining the weighted WAT fusion strategy with the objective search of $\alpha^{i}$ by SA has given the fusion method called WATSA.

\section{Data and study area}

The data used to evaluate this methodology correspond to two scenes recorded by the panchromatic and multispectral sensors onboard the IKONOS and QuickBird satellites. For the two scenes, the MULTI image size was $128 \times 128$ pixels, and consequently the PAN image size was $512 \times 512$ pixels. The IKONOS scene was recorded on 10 March 2000 and is geographically located in the Maipo Valley near Santiago, Chile. The QuickBird scene was extracted from an image registered on 22 August 2002, and the geographic area corresponds to the northwest area outside Madrid, Spain.

The PAN images of these scenes are presented in Figures 4a and $\mathbf{4 d}$, and their corresponding near-infrared-green-blue compositions of the MULTI image in Figures $4 \mathrm{~b}$ and $4 \mathrm{e}$.

Prior to the fusion process, source images should be preprocessed (Figure 3). The MULTI image must be resized to the PAN image size by an interpolation method, and it 


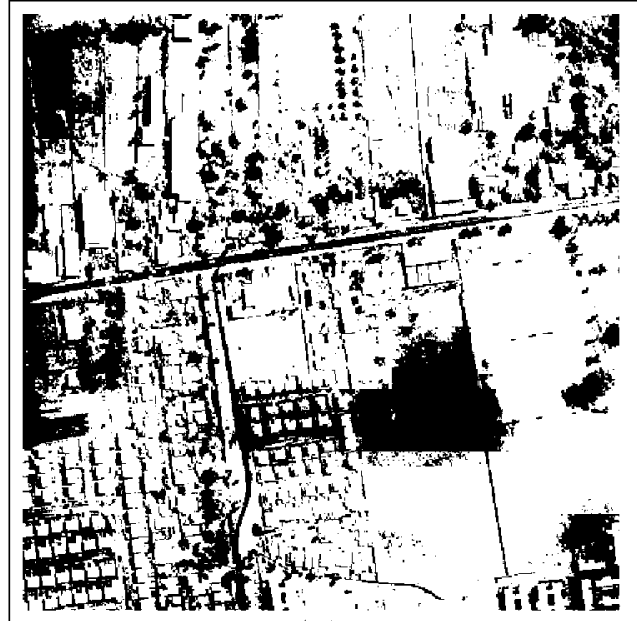

(a)

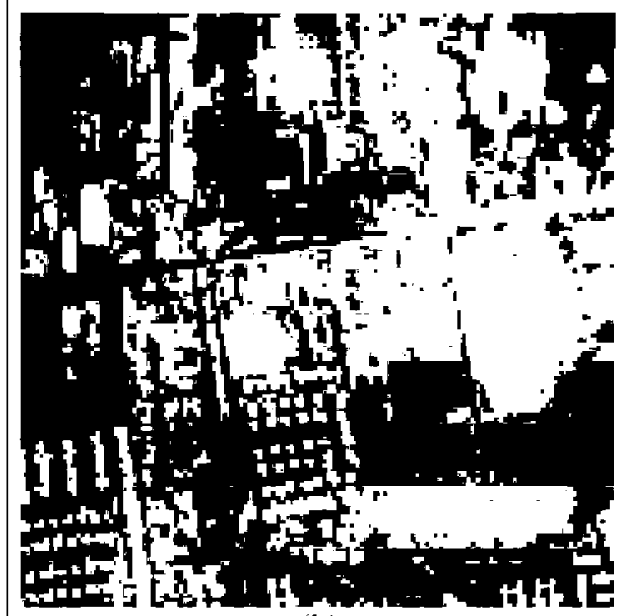

(b)

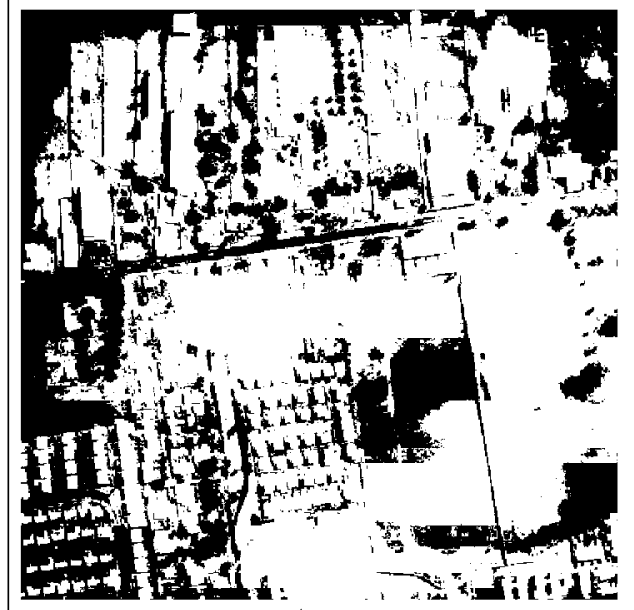

(c)

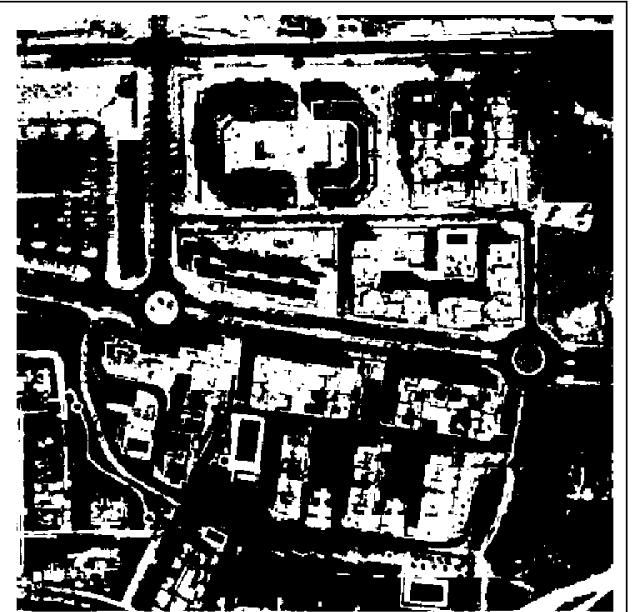

(d)

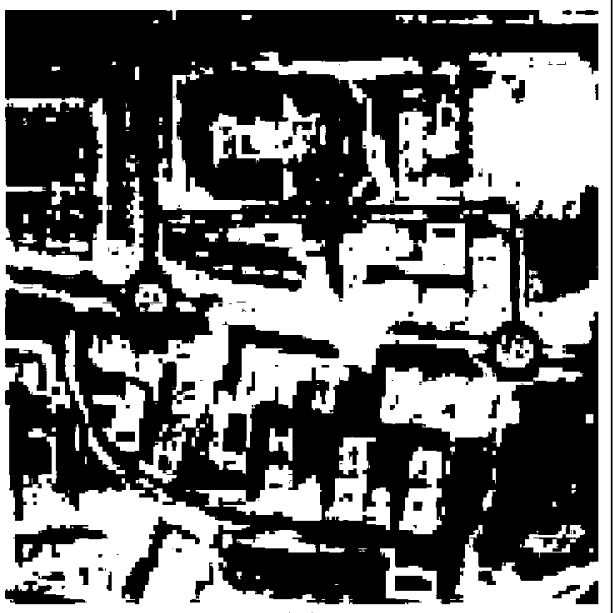

(e)

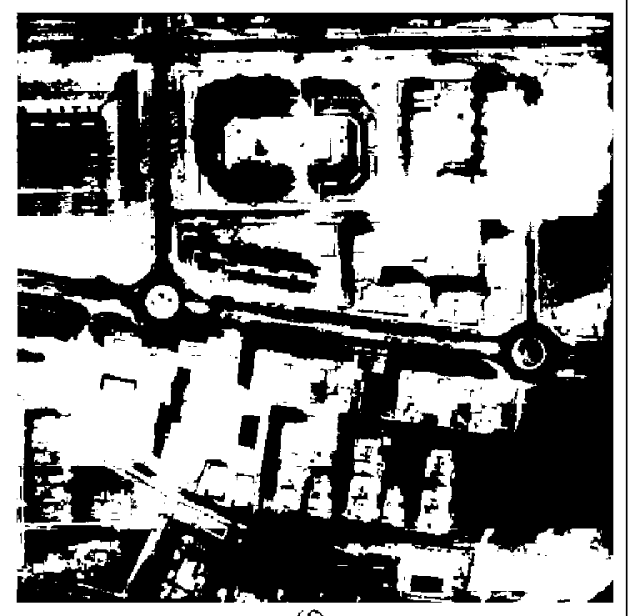

(f)

Figure 4. $(a-c)$ IKONOS scene. (d-f) QuickBird scene. PAN images (a and d), original MULTI images color composition (b and e), and WATSA fused images (c and f).

should also be coregistered with this last image. In this paper, data control points and a bicubic polynomial fit method (Núñez, 1999) have been used for coregistering the source images.

\section{Results and discussion}

To show the different behavior of the fusion strategies based on the standard WAT and the WATSA, the sources images (Figure 4) have been fused through both strategies for different 
values of the decomposition level $(n)$. In the first case, $\alpha^{i}=$ $100 \%$, and for the WATSA strategy the $\alpha^{i}$ values have been obtained by means of the directed search algorithm. These values have been included in Tables 1 and 2 . The differences between the $\alpha^{i}$ values justify the need to apply the search method for each band. Additionally, it is important to note the decrease that this factor suffers for each one of the bands when the decomposition level increases, as a consequence of the quality trade-off imposed by WATSA.

The indices ERGAS spatial $_{\text {ERGAS }}$ ERectral $_{\text {, and ERGAS }}$ average have been calculated for all fused images. Figures $\mathbf{5 a}$ and $\mathbf{5 b}$ present the variation of these indices with variation of the decomposition level $(n)$ for the WAT standard method. In these figures, it can be appreciated that the equality between

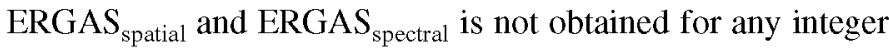
value of $n$; the decomposition level $n=2$ provides the best trade-off between both indices without being complete.

Figures 5c and 5d show variations in ERGAS indices with respect to $n$ for the WATSA strategy. There are two especially notable aspects in these figures, namely the preservation of the trade-off for all decomposition levels, and the existence of an $n$ value with minimum ERGAS values $(n=2$ for the IKONOS image $\left(\right.$ ERGAS $\left._{\text {average }}=1.8756\right)$ and $n=3$ for the QuickBird image $\left(E R G A S_{\text {average }}=1.7231\right)$ ). Figures $\mathbf{4} \mathbf{c}$ and $\mathbf{4 f}$ include the corresponding fused images, in which the spectral quality is conserved and the spatial quality is increased with respect to the original multispectral images.

Quality indices of the fused images provided by the WATSA method have been compared with the quality indices of fused images by two extensively used methods, namely the fast intensity-hue-saturation (FIHS) method (Tu et al., 2004) and the standard WAT fusion method. The quality indices evaluated
Table 1. WATSA $\alpha^{i}$ values for the IKONOS scene.

\begin{tabular}{lllll}
\hline Level & Band 1 & Band 2 & Band 3 & Band 4 \\
\hline 1 & 1.4523 & 1.1849 & 1.1929 & 1.3060 \\
2 & 0.8375 & 0.6827 & 0.6760 & 0.6216 \\
3 & 0.7124 & 0.5931 & 0.5800 & 0.4509 \\
4 & 0.6478 & 0.5478 & 0.5268 & 0.3511 \\
5 & 0.6009 & 0.5148 & 0.4861 & 0.2879 \\
6 & 0.5628 & 0.4882 & 0.4532 & 0.2484 \\
7 & 0.5303 & 0.4662 & 0.4263 & 0.2243 \\
8 & 0.5022 & 0.4476 & 0.4046 & 0.2114 \\
9 & 0.4776 & 0.4321 & 0.3873 & 0.2060 \\
10 & 0.4560 & 0.4190 & 0.3737 & 0.2056 \\
\hline
\end{tabular}

Table 2. WATSA $\alpha^{i}$ values for the QuickBird scene.

\begin{tabular}{lllll}
\hline Level & Band 1 & Band 2 & Band 3 & Band 4 \\
\hline 1 & 2.2929 & 1.7964 & 1.7299 & 2.0770 \\
2 & 1.0487 & 0.8117 & 0.7708 & 0.9124 \\
3 & 0.7793 & 0.6186 & 0.5853 & 0.6590 \\
4 & 0.6487 & 0.5286 & 0.4977 & 0.5330 \\
5 & 0.5615 & 0.4688 & 0.4384 & 0.4466 \\
6 & 0.4981 & 0.4251 & 0.3945 & 0.3813 \\
7 & 0.4519 & 0.3936 & 0.3625 & 0.3333 \\
8 & 0.4196 & 0.3722 & 0.3407 & 0.3008 \\
9 & 0.3983 & 0.3592 & 0.3272 & 0.2812 \\
10 & 0.3853 & 0.3525 & 0.3201 & 0.2710 \\
\hline
\end{tabular}

have been the spatial Zhou index (Zhou et al., 1998), the spectral correlation index (CC) (Vijarayaj et al., 2004), and the global indices SSIM (Wang et al., 2003) and ERGAS (Equation (5)). Since the Zhou, CC, and SSIM indices are

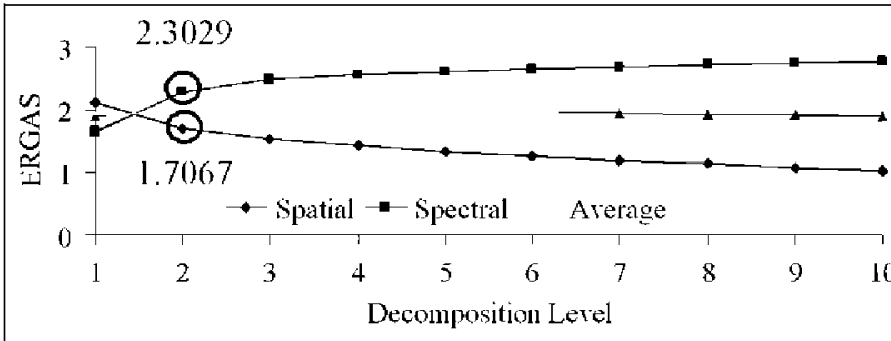

(a)

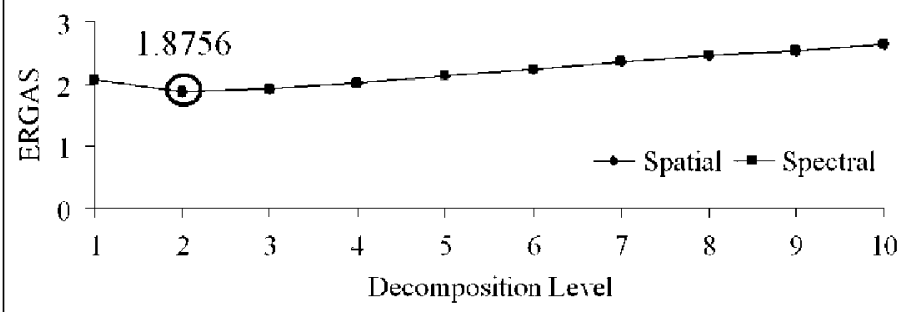

(c)

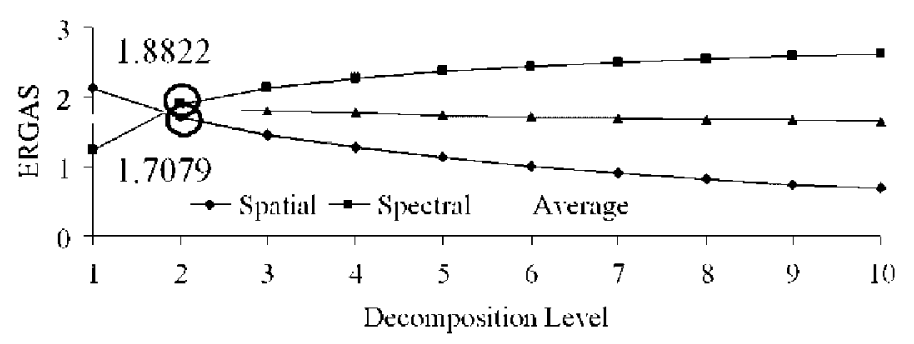

(b)

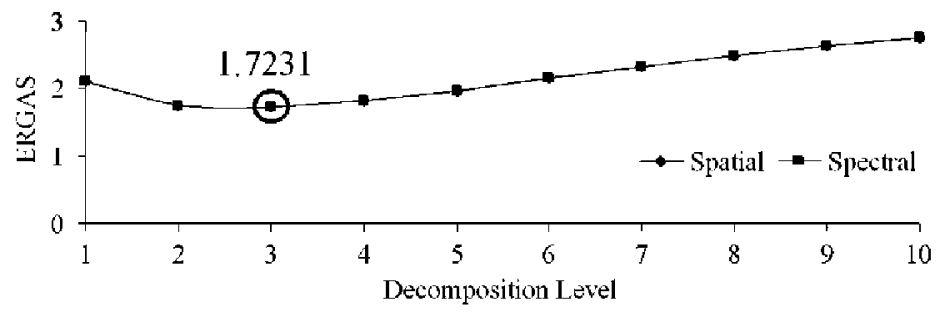

(d)

Figure 5. Variation of ERGAS indices with respect to decomposition level for $(a, b)$ the WAT-based fusion method with $2=100 \%$ for the IKONOS and QuickBird scenes, and (c, d) the WATSA fusion method for the IKONOS and QuickBird scenes. 
Table 3. IKONOS scene quality indices.

\begin{tabular}{lllll}
\hline Method & ERGAS $_{\text {spectral }}$ & Zhou & CC & SSIM \\
\hline FIHS & 1.8367 & 0.9707 & 0.8251 & 0.9838 \\
WAT & 2.0199 & 0.9940 & 0.8221 & 0.9837 \\
WATSA & 1.4892 & 0.9953 & 0.8879 & 0.9904 \\
\hline
\end{tabular}

Table 4. QuickBird scene quality indices.

\begin{tabular}{lllll}
\hline Method & ERGAS $_{\text {spectral }}$ & Zhou & CC & SSIM \\
\hline FIHS & 1.7844 & 0.9456 & 0.8449 & 0.9821 \\
WAT & 1.5094 & 0.9890 & 0.8845 & 0.9869 \\
WATSA & 1.3948 & 0.9946 & 0.9849 & 0.9885 \\
\hline
\end{tabular}

based on the correlation concept, the quality of the fused image is higher for high index values. The SSIM index has been computed with the parameters proposed by its authors. For computing the ERGAS $S_{\text {spectral }}$ index, the reference image used has been the original MULTI image, which imposes the resize of the fused image to the size of the reference image (original MULTI image). This has been carried out by the nearest neighbor method.

The index values calculated for the two scenes considered are summarized in Tables 3 and 4 . First, it can be observed that for the two scenes the WATSA fusion method provides the best index values, independently of their spectral and spatial character, lower values of the ERGAS spectral index, and higher values of the Zhou, CC, and SSIM indices than the other compared methods. However, the FIHS method provides better spectral quality than the WAT method for the IKONOS scene but lower spatial quality. For the Quickbird scene, both spatial and spectral qualities of the image fused by the WAT method are higher than those for the FIHS method.

\section{Conclusions}

The fusion method investigated in this paper (WATSA) has allowed us to obtain fused images with high spatial and spectral quality and optimal trade-off between both qualities. The main difference between the WATSA method and the previous weighting version has been the introduction of the directed searching, in an accurate and objective way, for the weighting factor $\alpha^{i}$ to be applied to the detailed panchromatic image information that will be integrated with the background multispectral image information, instead of the graphic method proposed in the previous version. The directed search has been carried out by the simulated annealing (SA) algorithm.

The main advantage of the new tune-up method resides in its exploratory character, which provides a noteworthy reduction in computing time to determine the weighting factor compared with that for the graphical method, since a lower number of fused images should be generated. On the other hand, that reduction has permitted more in-depth research to be conducted into the behavior of the weighting fusion strategy. However, it should be noted that for very large images the tune-up process can take a long time, and therefore it is necessary to establish a trade-off between computational cost and final quality. This trade-off is controlled by the temperature-decreasing index ( $\delta$ ).

Based on the results, it is concluded that the $\alpha^{i}$ values depend on the spectral band, decomposition level, and characteristics of the source images compared to other methods that only consider the spectral band dependence. This allows adapting the amount of information coming from the image PAN that should be injected in the MULTI image, for each particular source image. However, it should also be considered that for large images or images with large numbers of land covers a unique value of the weighting factor for each spectral band could not provide an improved solution versus the standard WAT fusion method.

The $\alpha^{i}$ values obtained have provided fused images with an optimal trade-off between spatial and spectral qualities for any decomposition level. The existence of an $n$ value has also been shown that is dependent on image characteristics (sensor and coverage type), for which minimum ERGAS (spatial, spectral, and average) values are obtained. In other words, fused images with "the best" spatial and spectral quality can be obtained while an optimal trade-off between both qualities is maintained.

The evaluation of different quality indices, based on correlation coefficients (Zhou, CC, and SSIM) and RMSE

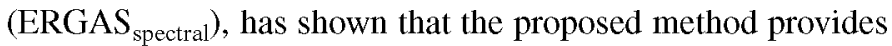
not only a trade-off between the spatial and spectral quality of the fused images, but also higher spatial and spectral qualities than those for the other investigated methods.

\section{Acknowledgements}

This work has been jointly supported by Universidad Politécnica de Madrid, Spain (AL08-P(I+D)-19), the Spanish Government (TEC2007-60607/TCM), and the Chile Research Council (FONDECYT 11060056). The authors would like to thank INDRA-Espacio for facilitating the QuickBird image used in this study.

\section{References}

Candès, E.J., and Donoho, D.L. 2000. Curvelets, multiresolution representation, and scaling laws. In Wavelet Applications in Signal and Image Processing VIII. Edited by A. Aldroubi, A.F. Laine, and M.A. Unser. The International Society for Optical Engineering (SPIE), Bellingham, Wash. Proceedings of SPIE Volume 4119, pp. 1-12.

Chibani, Y., and Houacine, A. 2003. Redundant versus orthogonal wavelet decomposition for multisensor image fusion. Pattem Recognition, Vol. 36, pp. $879-887$.

Choi, M. 2006. A new intensity-hue-saturation fusion approach to image fusion with a tradeoff parameter. IEEE Transactions on Geoscience and Remote Sensing, Vol. 44, pp. 1672-1682.

Choi, M., Kim, R., Nam, M., and Kim, H. 2005. Fusion of multispectral and panchromatic satellite images using the Curvelet transform. IEEE Geoscience and Remote Sensing Letters, Vol. 2, pp. 136-140. 
Dutilleux, P. 1987. An implementation of the algorithm à trous to compute the wavelet transform. In Compt-rendus du congrès ondelettes et méthodes temps-fréquence et espace des phases. Edited by J. Combes, A. Grossmann, and Ph. Tchanitchian. Springer-Verlag, Marseille. pp. 298-304.

González-Audicana, M., Otazu, X., Fors, O. and Seco, A. 2005. Comparison between the Mallat's and the à trous discrete wavelet transform based algorithms for the fusion of multispectral and panchromatic image. International Joumal of Remote Sensing, Vol. 26, pp. 597-616.

Kirkpatrick, S., Gelatt, C.D., and Vecchi, M.P. 1983. Optimization by simulated annealing. Science (Washington, D.C.), Vol. 4598, pp. 671-680.

Lillo-Saavedra, M., and Gonzalo, C. 2006a. Spectral or spatial quality for fused satellite imagery? A trade-off solution using wavelet à trous algorithm. International Joumal of Remote Sensing, Vol. 27, pp. 14531464.

Lillo-Saavedra, M., and Gonzalo, C. 2006b. Objective determination of the degradation level of the multispectral and panchromatic images for fusion by means of the à trous algorithm. In Global Developments in Environmental Earth Observation from Space, Proceedings of the Symposium of the European Association of Remote Sensing Laboratories, 25 June 2006, Porto, Portugal. Edited by A. Marcal. Millpress Science Publishers, Rotterdam, The Netherlands. pp. 103-110.

Lillo-Saavedra, M., and Gonzalo, C. 2007. Multispectral images fusion by a joint multidirectional and multiresolution representation. International Journal of Remote Sensing, Vol. 28, pp. 4065-4079.

Lillo-Saavedra, M., Gonzalo, C., Arquero A., and Martínez, E. 2005. Fusion of multispectral and panchromatic satellite sensor imagery based on tailored filtering in the Fourier domain. International Joumal of Remote Sensing, Vol. 26, pp. 1263-1268.

Mallat, S. 1999. A wavelet tour of signal processing. 2nd ed. Academic Press, San Diego, Calif. Chapt. 7, Sect. 3, p. 255.

Núñez, J., Otazu, X., Fors, O., Prades, A., Palá, V., and Arbiol, R. 1999. Multiresolution-based image fusion with additive wavelet decomposition. IEEE Transactions on Geoscience Remote Sensing, Vol. 37, pp. 1204 1211 .

Tu, T.M., Huang, P.S., Hung, C.L., and Chang, C.P. 2004. A fast intensityhue-saturation fusion technique with spectral adjustment for IKONOS imagery. IEEE Geoscience and Remote Sensing Letters, Vol. 1, pp. 309 312.

Tu, T.M., Cheng, W.C., Chang, C.P., Huang, P.S., and Chang, J.C. 2007. Best tradeoff for high-resolution image fusion to preserve spatial details and minimize color distortion. IEEE Transactions on Geoscience and Remote Sensing, Vol. 4, pp. 302-306.

Vijayaraj, V., O'Hara, C.G., and Younan, N.H. 2004. Quality analysis of pansharpened images. In IGARSS'04, Proceedings of the International Geosciences and Remote Sensing Symposium, 20-24 September 2004, Anchorage, Alaska. IEEE, Piscataway, N.J. pp. 85-88.

Wald, L. 2002. Data fusion, definition and architectures: fusion of image of different spatial resolution. Le Presses de l'École des Mines, Paris.

Wang, Z., Simoncelli, E.P., and Bovik, A.C. 2003. Multi-scale structural similarity for image quality assessment. In Proceedings of the 37th IEEE Asilomar Conference on Signal Systems and Computers, 9-12 November 2003, Pacific Grove, Calif.

Yunhao, C., Lei, D., Jing, L., Xiaobing, L., and Peijun, S. 2006. A new wavelet-based image fusion method for remotely sensed data. International Joumal of Remote Sensing, Vol. 27, pp. 1465-1476.
Zhou, J., Civco, D.L., and Silander, J.A. 1998. A wavelet method to merge Landsat TM and SPOT panchromatic data. International Journal of Remote Sensing, Vol. 19, pp. 743-757. 